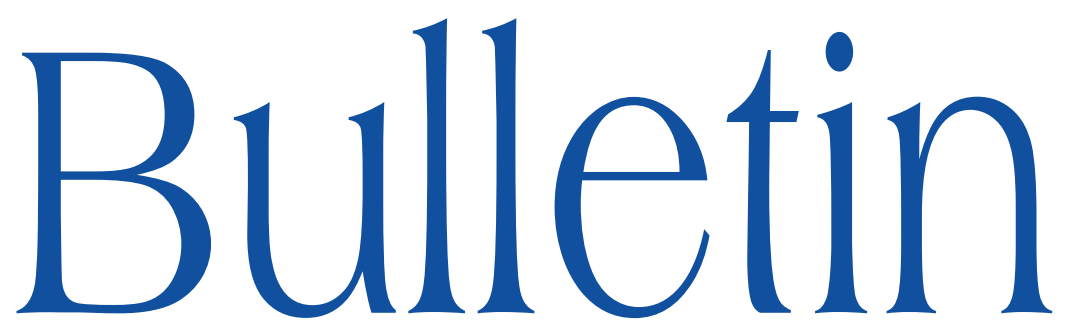

de la SOCIÉTÉ MATHÉMATIQUE DE FRANCE

\title{
TRANSFORMÉE DE RADON SEMI-GLOBALE
}

\author{
Mehdi Benchoufi
}

Tome 139

Fascicule 2

2011

SOCIÉTÉ MATHÉMATIQUE DE FRANCE

Publié avec le concours du Centre national de la recherche scientifique 


\title{
TRANSFORMÉE DE RADON SEMI-GLOBALE
}

\author{
PAR MEHDi BENCHOUFI
}

\begin{abstract}
RÉsumé. - Dans cet article, nous nous proposons d'étudier le noyau, l'image et une éventuelle formule d'inversion de la transformation de Radon réelle dans les domaines linéairement concaves. Nous rappelons que, dans $\mathbb{R}^{2}$, on sait reconstruire une fonction à partir de sa transformation de Radon lorsque celle-ci est connue le long de toutes les droites de l'espace. Notre propos sera, en quelque sorte, d'établir une version semiglobale de ce résultat. Nous verrons ainsi que, modulo un noyau que nous préciserons, constitué de sauts de fonctions holomorphes, chacune définie sur un « wedge » et vérifiant dans leurs domaines respectifs une majoration en $\theta\left(\frac{1}{|z|^{2}}\right)$ lorsque $|z|$ tend vers l'infini, une formule d'inversion est accessible dès lors que la transformation de Radon n'est connue qu'au voisinage d'une droite.
\end{abstract}

Abstract (Semi-global Radon Transformation). - In this article, we mean to study the kernel, the image and a possible inversion formula for the real Radon transform in linearly concave domains. We recall that, in $\mathbb{R}^{2}$, we know how to reconstruct a function from its Radon transform when the latest is known all along every lines of the space. Our purpose will be somehow to establish a semi-global analogue of this result. In this way, we will see that, modulo a kernel we will precise, consisting of jumps of holomorphic functions, each of which is defined upon a "wedge" and submitted to an estimation in $\Theta\left(\frac{1}{|z|^{2}}\right)$ when $|z|$ tends to infinity, an inversion formula is reachable as soon as the Radon transform is known in the neighbourhood of a line.

Texte reçu le 31 juillet 2006, révisé les 20 mars 2009 et 27 juillet 2009, accepté le 21 septembre 2009

Mendi Benchoufi, Université Pierre et Marie Curie, Paris VI, Institut de Mathématiques de Jussieu, 175, rue du Chevaleret 75013 Paris • E-mail : benchoufi@math.jussieu.fr Classification mathématique par sujets (2000). — 32A26, 44A12.

Mots clefs. - Transformée de Radon, semi-globale, analyse complexe. 


\section{Introduction}

Commençons par définir la transformation de Radon réelle.

DÉFInition 1.1 (Funk [3], Radon [12]). — On représente un hyperplan dans $\mathbb{R}^{n}$ par l'équation : $\left\{x \in \mathbb{R}^{n} \mid\langle\theta, x\rangle=t\right\}$ où $\theta \in S^{n-1}=\left\{\theta \in \mathbb{R}^{n}|| \theta \mid=1\right\}$ et $t \in \mathbb{R}$. Pour toute fonction $f$, de $\mathbb{R}^{n}$ dans $\mathbb{R}$, intégrable sur tous les hyperplans de $\mathbb{R}^{n}$, et pour tous $(\theta, t) \in S^{n-1} \times \mathbb{R}$, on définit la transformation de Radon réelle $(R f)_{\mathbb{R}}(\theta, t)$ par la formule suivante :

$$
\left.(R f)_{\mathbb{R}}(\theta, t)=\int_{\langle\theta, x\rangle=t} f(x) \theta . d x\right\rfloor d x_{1} \wedge d x_{2} \wedge \cdots \wedge d x_{n}
$$

Ici $\theta . d x=\sum_{i=1}^{n} \theta_{i} d x_{i}$ et $\left.\theta . d x\right\rfloor d x_{1} \wedge d x_{2} \wedge \cdots \wedge d x_{n}$ désigne la forme $\omega$ telle que $\theta . d x \wedge \omega=d x_{1} \wedge d x_{2} \wedge \cdots \wedge d x_{n}$.

Dans cet article, on considèrera la restriction de cette transformation à un domaine linéairement concave, c'est-à-dire une réunion d'hyperplans de $\mathbb{R}^{n}$. Dans un esprit assez proche, on peut définir la transformation de Radon complexe. C'est ce qui amène à la définition suivante :

DÉFInition 1.2 (Martineau [11], Gindikin,Henkin [5] : Transformation de Radon complexe)

Soit $\mathrm{K}$ un compact linéairement convexe dans $\mathbb{C P}^{n}$ et $\Omega=\mathbb{C P}^{n} \backslash K, f \in$ $\mathscr{C}_{n, n-1}(\Omega)$, telle que $\bar{\partial} f=0$. Alors on définit la transformation de Radon complexe de $f$ par la formule suivante:

$$
(R f)_{\mathbb{C}}(\xi)=\frac{1}{2 \pi i} \int_{z \in \Omega} \bar{\partial}_{z}\left(\frac{\xi_{0} \cdot z_{0}}{\langle\xi, z\rangle}\right) \wedge f, \xi \in K^{*} .
$$

Ici $K^{*}$ désigne l'ensemble des éléments de $\mathbb{C} P^{n}$ tels que $\left\{z \in \mathbb{C P}^{n} \mid\langle\xi, z\rangle=\right.$ $\left.\xi_{0} z_{0}+\cdots+\xi_{n} z_{n}=0\right\} \subset \mathbb{C} P^{n} \backslash K$, avec $\xi=\left(\xi_{0}, \xi_{1}, \ldots, \xi_{n}\right)$ et $z=\left(z_{0}, z_{1}, \ldots, z_{n}\right)$. Et $\mathscr{C}_{n, n-1}(\Omega)$ désigne l'espace des $(n, n-1)$-formes continues sur $\Omega$.

Soit $\Omega$ un domaine de $\mathbb{R}^{n}$, et $\Omega^{*}$ son domaine dual, i.e. l'ensemble des hyperplans contenus dans $\Omega$. On dira que $\Omega$ est un domaine linéairement concave si par chacun de ses points passe un hyperplan contenu dans $\Omega$. On dira que $\Omega$ est un domaine fortement linéairement concave si par chacun de ses points passe un hyperplan contenu dans $\Omega$ et s'il existe une détermination $\mathscr{C}^{1}$ de ces hyperplans par les points de $\Omega$.

Un problème qui se pose alors naturellement est celui de la reconstitution de la fonction $f$ à partir de sa transformée de Radon réelle. Pour ce qui est du cas global, i.e. du cas où la transformée de Radon est connue le long de tous les hyperplans de l'espace $\mathbb{R}^{n}$, les résultats sur l'injectivité et la surjectivité relatifs à des espaces de départ et d'arrivée bien précis ont été obtenus successivement par Radon [12], John [9] et par Gelfand, Graev, Vilenkin [4], Helgason [6],.... 
Ainsi, lorsque la transformation de Radon d'une fonction est connue le long de l'ensemble des hyperplans de l'espace $\mathbb{R}^{n}$, il est possible de reconstituer la valeur de cette même fonction en chaque point via une formule découverte par Radon [12], qu'il est convenu d'appeler formule explicite d'inversion de Radon. On peut, après cela, se poser la question plus générale de l'existence d'une formule d'inversion dès lors que la transformation de Radon d'une fonction est seulement connue le long d'hyperplans contenus dans un domaine linéairement concave de l'espace $\mathbb{R}^{n}$.

Dans le cas complexe, et plus précisément, dans les domaines fortement linéairement concaves de $\mathbb{C P}^{n}$, le résultat suivant a été établi :

Théorème 1.3 (Gindikin, Henkin [5]; Henkin, Polyakov [8])

Soit $D$ un domaine fortement linéairement concave de $\mathbb{C P}^{n}$. Pour toute forme $f, \bar{\partial}$-fermée, appartenant à $\mathscr{C}_{0, n-1}^{1}(D)$, espace des $(0, n-1)$-formes de classe $\mathscr{C}^{1}$ sur $D$, il existe des opérateurs explicites $\Xi$ et $H$, qui permettent la représentation suivante :

$$
f=\Xi\left((R f)_{\mathbb{C}}\right)+\bar{\partial} H f
$$

(La définition de la concavité forte pour les domaines complexes est exactement similaire à celle des domaines réelles; il suffit de remplacer « hyperplan réel » par « hyperplan complexe»). C'est dans cet esprit qu'Henkin [7](Theo.5), a annoncé la possibilité de déduire de la formule précédente un résultat semblable pour la transformation de Radon réelle.

A présent, quelques notations et hypothèses utiles pour la suite du texte.

Dans cet article, nous n'envisagerons que le cas $n=2$.

Désignons d'abord par $\Omega$ un domaine fortement linéairement concave de $\mathbb{R}^{2}$, distinct de $\mathbb{R}^{2}$, et par $\Omega^{*}$ l'ensemble des droites, paramétrées par un sousensemble de $S^{1} \times \mathbb{R}$, contenues dans $\Omega$. Un élément $(\theta, t)$ de $S^{1} \times \mathbb{R}$ paramétrisera la droite $\left\{\left(x_{1}, x_{2}\right) \in \mathbb{R}^{2} \mid t+\theta_{1} x_{1}+\theta_{2} x_{2}=0\right\}$. Nous noterons $\pi\left(\Omega^{*}\right)$, la projection de $\Omega^{*}$ sur $S^{1}$. De manière plus uniforme, nous pourrions choisir de présenter $\Omega$, comme le complémentaire d'un compact convexe non vide de $\mathbb{R P}^{2}$.

Par ailleurs, dans notre article, nous serons amené à utiliser un ensemble $\Omega_{\mathbb{C}}$, construit en complexifiant chacune des droites contenues dans $\Omega$, tel que $\Omega \subset \Omega_{\mathbb{C}} \subset \mathbb{C P}^{2}$. Nous noterons alors $\Omega_{ \pm}$les deux composantes connexes de $\Omega_{\mathbb{C}} \backslash \Omega$.

Nous noterons $\mathscr{C}^{n, m}\left(\mathbb{R}^{2}, \mathbb{C}\right), n, m \in \mathbb{N}$, la classe des fonctions $f$ de $\mathbb{R}^{2}$ à valeurs dans $\mathbb{C}$, appartenant à $\mathscr{C}^{n}\left(\mathbb{R}^{2}, \mathbb{C}\right)$, telles que

$$
\sup _{x \in \mathbb{R}^{2}}(1+|x|)^{m}\left|\frac{\partial^{k}}{\partial x_{1}^{i} \partial x_{2}^{j}} f(x)\right|<\infty .
$$

$k=0,1, \ldots, m ; i+j=k$. 
Le plan de Henkin [7] consisterait à montrer que, pour un domaine $\Omega$ linéairement concave de $\mathbb{R}^{2}$, et pour une fonction $f$, il existe un opérateur $B_{\mathbb{R}}$, à valeur dans l'espace des fonctions continues de $\Omega$ dans $\mathbb{C}$ et des opérateurs $K_{ \pm}$ à valeurs dans l'espace des fonctions holomorphes dans $\Omega_{ \pm}$, vérifiant dans leurs domaines respectifs une majoration en $\Theta\left(\frac{1}{|z|^{2}}\right)$ lorsque $|z|$ tend vers l'infini, qui permettent la décomposition

$$
f=B\left((R f)_{\mathbb{R}}\right)+K_{+} f+K_{-} f .
$$

Le but de cet article n'est pas seulement de démontrer l'existence de tels opérateurs mais d'en donner des formules explicites. Les formules que nous nous proposons d'exhiber peuvent recevoir des applications en tomographie.

Nous nous proposons donc d'étudier la transformation de Radon réelle dans des domaines linéairement concaves. Plus précisément, on cherche d'abord à obtenir une caractérisation de son noyau. On montrera que ce dernier consiste en des sauts de fonctions holomorphes, définies sur des « wedge » que nous préciserons par la suite et vérifiant dans leurs domaines respectifs une majoration en $\Theta\left(\frac{1}{|z|^{2}}\right)$ lorsque $|z|$ tend vers l'infini. Ceci nous amènera au résultat annoncé par Henkin dans [7] :

THÉORÈme 1.4. - Soit $f$ appartenant à $\varrho^{1}\left(\mathbb{R}^{2}, \mathbb{C}\right)$ et intégrable sur les droites de $\Omega^{*}$. On suppose que la transformation de Radon réelle s'annule sur l'ensemble des droites d'un domaine fortement linéairement concave $\Omega$. Alors $f$ se décompose en la différence de 2 fonctions holomorphes définies respectivement dans les domaines $\Omega_{ \pm}$, et vérifiant dans leurs domaines respectifs une majoration du type $f_{ \pm}(z)=\Theta\left(\frac{1}{|z|^{2}}\right)$ lorsque $|z|$ tend vers l'infini. On a

$$
\begin{gathered}
f_{ \pm}(z)= \\
\left.\left.-\frac{1}{2 \pi i} \int_{\langle\vartheta(z), \xi-z\rangle=0, \Im(\xi)=\vartheta(z)^{\perp}} f(\Re(\xi)) \operatorname{det}\left(\vartheta(z), \frac{\Im(\xi)}{\langle\Im(\xi), \Re(\xi)-z\rangle}\right)(\vartheta(z) d \xi)\right\rfloor \omega(\xi)\right) \\
\text { pour } z \in \Omega_{ \pm} .
\end{gathered}
$$

Ici $\omega$ désigne la 2-forme $\omega(\xi)=d \xi_{1} \wedge d \xi_{2}$ et $\vartheta$ est une application continue, dite section de Leray, du domaine $\Omega_{\mathbb{C}}$ dans $S^{1}$, que nous construirons dans la partie 2. On note $\langle\vartheta(z), \xi-z\rangle=\vartheta_{1}(z)\left(\xi_{1}-z_{1}\right)+\vartheta_{2}(z)\left(\xi_{2}-z_{2}\right)$. Nous noterons $\vartheta(z)^{\perp}$ l'élément $\left(-\vartheta_{2}(z), \vartheta_{1}(z)\right)$. Nous verrons que $\vartheta$ sera choisie de manière particulière : le domaine d'intégration $\left\{\xi \in \mathbb{C}^{2} \mid\langle\vartheta(z), \xi-z\rangle=0, \Im(\xi)=\right.$ $\left.\frac{\vartheta(z)^{\perp}}{\left|\vartheta(z)^{\perp}\right|}\right\}$ sera alors constitué d'une droite réelle 《pointée » en un élément du plan imaginaire.

C'est au cours de la démonstration de ce théorème que nous démontrerons la proposition fondamentale suivante, qui rapporte le $\bar{\partial}$ de $f_{ \pm}$et la transformation de Radon de $f$. 
Proposition 1.5. - Nous avons, pour $z \in \Omega_{ \pm}$, l'identité suivante

$$
\begin{aligned}
\left(\vartheta_{1}(z) \bar{\partial}_{z_{1}}+\right. & \left.\vartheta_{2}(z) \bar{\partial}_{z_{2}}\right) f_{ \pm}(z) \\
& =\frac{i}{|\Im(z)|}\left(\vartheta_{1}(z) \frac{\partial}{\Re\left(z_{1}\right)}+\vartheta_{2}(z) \frac{\partial}{\Re\left(z_{2}\right)}\right)(R f)_{\mathbb{R}}(\vartheta(z),\langle\vartheta(z), \Re(z)\rangle) .
\end{aligned}
$$

où $|\Im(z)|$ désigne $\left(\Im\left(z_{1}\right)^{2}+\Im\left(z_{2}\right)^{2}\right)^{\frac{1}{2}}$.

Le théorème 1.4. peut être vu comme une précision du théorème de régularité micro-locale de Boman [1]. Ce dernier utilise essentiellement des outils micro-locaux, à savoir un théorème de régularité micro-locale des solutions de l'équation $R(f)_{\mathbb{R}}=0$.

Par ailleurs, nous établirons une caractérisation de l'image de la transformation de Radon :

ThÉORÈme 1.6. - Soit $g \in \mathscr{C}^{3,2}\left(\bar{\Omega}^{*}, \mathbb{C}\right)$, telle que $g$ vérifie $g(\theta, t)=$ $g(-\theta,-t)$, alors il existe $f \in \mathscr{C}^{1}(\Omega, \mathbb{C})$, intégrable sur toutes les droites de $\Omega^{*}$, telle que $(R f)_{\mathbb{R}}=g$.

où $\mathscr{C}^{3,2}\left(\bar{\Omega}^{*}, \mathbb{C}\right)$ désigne les fonctions de $\mathscr{C}^{3}\left(\bar{\Omega}^{*}, \mathbb{C}\right)$, et vérifiant, uniformément par rapport à $\theta \in \pi\left(\bar{\Omega}^{*}\right)$ et pour $i+j=0,1,2,3, \frac{d^{i}}{d \theta^{i}} \frac{d^{j}}{d t^{j}} g(\theta, t)=\emptyset\left(\frac{1}{t^{2}}\right)$ quand $t$ tend vers l'infini. Ici $\bar{\Omega}^{*}$ désigne la fermeture de $\Omega^{*}$. La version globale de ce résultat, à savoir le cas où $\Omega=\mathbb{R}^{2}$, est due à Radon [12].

Enfin, nous verrons dans la démonstration du théorème qui suit, qu'étant donnée la transformation de Radon d'une fonction $f$ dans un domaine fortement linéairement concave $\Omega$, l'on peut construire $\overline{R(f)_{\mathbb{R}}}$ correspondant à une extension à $S^{1} \times \mathbb{R}$ de $R(f)_{\mathbb{R}}$ définie sur $\Omega^{*}$. La construction de cette extension sera explicitement présenté dans la démonstration du théorème qui suit. On a le théorème suivant :

THÉORÈme 1.7. - Soit $f$ une fonction appartenant à $\mathscr{C}_{c}^{3}\left(\mathbb{R}^{2}, \mathbb{C}\right)$. On ne suppose plus que la transformation de Radon réelle s'annule dans le domaine fortement linéairement concave $\Omega$. Alors $f$ s'écrit comme la somme

$$
f=B(R f)_{\mathbb{R}}+K_{+} f+K_{-} f .
$$

$K_{+} f+K_{-} f$ est la somme de fonctions respectivement holomorphes dans les domaines $\Omega_{+}$et $\Omega_{-}$, et vérifiant dans leurs domaines respectifs une majoration du type $K_{ \pm} f(z)=\theta\left(\frac{1}{|z|^{2}}\right)$ lorsque $|z|$ tend vers l'infini. La somme $K_{+} f+K_{-} f$ est entendu au sens où, si $x$ est un élément de $\Omega$, et si l'on se donne deux suites quelconques d'éléments $(z)_{+}$de $\Omega_{+}$et $(z)_{-}$de $\Omega_{-}$convergeant toutes deux vers $x$, alors $K_{+} f(x)+K_{-} f(x)$ désigne $\lim _{(z)_{ \pm} \rightarrow x}\left(K_{+} f\left(z_{+}\right)+K_{-} f\left(z_{-}\right)\right)$. 
Plus précisément, pour $x \in \Omega$,

$$
B\left(R(f)_{\mathbb{R}}\right)(x)=\frac{1}{(2 \pi i)^{2}} \int_{t \in \mathbb{R}} \int_{\theta \in S^{1}} \frac{\overline{R(f)_{\mathbb{R}}}(\theta, t)}{(t-\langle\theta, x\rangle+i 0)^{2}} \omega^{\prime}(\theta) \wedge d t
$$

ò̀ $\omega^{\prime}(\theta)=\theta_{2} d \theta_{1}-\theta_{1} d \theta_{2}$, et

$$
\begin{gathered}
K_{ \pm} f(z)=-\frac{1}{2 \pi i} \int_{\langle\vartheta(z), \xi-z\rangle=0, \Im(\xi)=\vartheta(z)^{\perp}} \\
\left.\left.\left(f(\Re(\xi))-B\left(R(f)_{\mathbb{R}}\right)(\Re(\xi))\right) \operatorname{det}\left(\vartheta(z), \frac{\Im(\xi)}{\langle\Im(\xi), \Re(\xi)-z\rangle}\right)(\vartheta(z) d \xi)\right\rfloor \omega(\xi)\right)
\end{gathered}
$$

De plus, la transformation de Radon $R\left(K_{+} f+K_{-} f\right)_{\mathbb{R}}$ s'annule.

Nous remarquons que, lorsque $\Omega=\mathbb{R}^{2}$, cette formule nous donne bien la formule classique d'inversion de Radon globale [12].

\section{Noyau de la transformation de Radon dans les domaines linéairement concaves de $\mathbb{R}^{2}$}

2.1. Application de la formule de Sohotski-Plemelj. - Pour $x=\left(x_{1}, x_{2}\right) \in \Omega$, on note

$$
\Omega_{x}^{*}=\left\{(\theta, t) \in \Omega^{*} \mid\left\{y \in \mathbb{R}^{2} \mid t+\theta_{1} y_{1}+\theta_{2} y_{2}=0\right\} \subset \Omega, t+\theta_{1} x_{1}+\theta_{2} x_{2}=0\right\} .
$$

Pratiquement, pour chaque $x$, on associe le paramétrage de l'ensemble des droites passant par $x$ et incluses dans $\Omega$.

On note $\Omega_{\mathbb{C}}=\cup_{(\theta, t) \in \Omega^{*}}\left\{z \in \mathbb{C}^{2}, t+\theta_{1} z_{1}+\theta_{2} z_{2}=0\right\}$. Nous noterons alors $\Omega_{ \pm}$les deux composantes connexes de $\Omega_{\mathbb{C}} \backslash \Omega$.

A tout élément $z=\left(z_{1}, z_{2}\right)$ de $\Omega_{\mathbb{C}} \backslash \Omega$, on associe l'élément $\vartheta(z)=$ $\left(\vartheta_{1}(z), \vartheta_{2}(z)\right)$ de $S^{1},\left(\frac{\Im\left(z_{2}\right)}{|\Im(z)|},-\frac{\Im\left(z_{1}\right)}{|\Im(z)|}\right)$, coefficient directeur de la droite complexe passant par $\left(z_{1}, z_{2}\right)$ et par $\left(\Re\left(z_{1}\right), \Re\left(z_{2}\right)\right)$. La droite, $\left\{\xi \in \mathbb{C}^{2} \mid\langle\vartheta(z), \xi-z\rangle=0\right\}$, est ainsi entièrement contenue dans $\Omega_{\mathbb{C}}$.

$\Omega$ étant fortement linéairement concave, on a aussi supposé qu'il existait une application paramétrant de manière $\mathscr{C}^{1}$ les droites de $\Omega$ par les points de $\Omega$. On note aussi $\vartheta$ l'application $\zeta^{1}$ de $\Omega$ dans $\Omega^{*}$ telle que tout élément $x$ de $\Omega$ appartient à son image dans $\Omega^{*}$ par $\vartheta$ (image paramétrée par un élément de $S^{1}$ noté encore $\left.\vartheta(x)\right)$.

Pour une fonction $h$ de $\mathbb{R}$, intégrable à valeurs complexes, la formule de Plemelj -Sohotski donne, pour $x \in \mathbb{R}$ :

$$
h(x)=\lim _{\varepsilon \rightarrow 0}\left(\frac{1}{2 \pi i} \int_{y \in \mathbb{R}} \frac{h(y)}{y-x+i \varepsilon} d y-\frac{1}{2 \pi i} \int_{y \in \mathbb{R}} \frac{h(y)}{y-x-i \varepsilon} d y\right)
$$


Cette formule est l'application de la formule de Cauchy à une variable, quand on a plongé naturellement $\mathbb{R}$ dans $\mathbb{C}$.

Soit $f$ une fonction appartenant à $\mathscr{C}^{1}\left(\mathbb{R}^{2}, \mathbb{C}\right)$ et intégrable sur les droites de $\Omega^{*}$. Soit $x \in \Omega$. En appliquant cette formule selon chacune des droites réelles $\Re\left(\left\{\xi \in \mathbb{C}^{2} \mid\langle\vartheta(x), \xi-x\rangle=0\right\}\right)$ plongées dans la droite complexe $\{\xi \in$ $\left.\mathbb{C}^{2} \mid\langle\vartheta(x), \xi-x\rangle=0\right\}$. On obtient alors :

(2) $f(x)=\lim _{\varepsilon \rightarrow 0}\left(\frac{1}{2 \pi i} \int_{\langle\vartheta(x), \xi-x\rangle=0, \Im(\xi)=0} \frac{f(\Re(\xi))}{\left\langle\vartheta(x)^{\perp}, \Re(\xi)-x\right\rangle+i \varepsilon}(\vartheta(x) d \xi)\right\rfloor \omega(\xi)$

$$
\left.\left.-\frac{1}{2 \pi i} \int_{\langle\vartheta(x), \xi-x\rangle=0, \Im(\xi)=0} \frac{f(\Re(\xi))}{\left\langle\vartheta(x)^{\perp}, \Re(\xi)-x\right\rangle-i \varepsilon}(\vartheta(x) d \xi)\right\rfloor \omega(\xi)\right)
$$

En effet, ceci représente exactement la formule de Plemelj -Sohotski. Il suffit de faire le changement de variable $\xi_{1}^{\prime}:=\left\langle\vartheta(x)^{\perp}, \xi-x\right\rangle$ et $\xi_{2}^{\prime}:=\langle\vartheta(x), \xi-x\rangle$. Ici, $\vartheta(x)^{\perp}$ désigne l'orthogonal de $\vartheta(x)$.

Nous allons transformer cette expression pour rendre sa manipulation plus commode, comme nous le verrons dans la partie 2.2.

En effet, si l'on désigne par $f_{ \pm}$les fonctions définies sur $\Omega_{ \pm}$telles que, pour $z \in \Omega_{ \pm}:$

$$
\begin{gathered}
f_{ \pm}(z)=-\frac{1}{2 \pi i} \int_{\langle\vartheta(z), \xi-z\rangle=0, \Im(\xi)=\vartheta(z)^{\perp}} \\
\left.\left.f(\Re(\xi)) \operatorname{det}\left(\vartheta(z), \frac{\Im(\xi)}{\langle\Im(\xi), \Re(\xi)-\Re(z)\rangle-i\langle\Im(\xi), \Im(z)\rangle}\right)(\vartheta(z) d \xi)\right\rfloor \omega(\xi)\right)
\end{gathered}
$$

alors, la formule (2) s'écrit, pour $x \in \Omega$ :

$$
f(x)=\lim _{\varepsilon \rightarrow 0}\left(f_{+}\left(x+i \varepsilon \vartheta(x)^{\perp}\right)-f_{+}\left(x-i \varepsilon \vartheta(x)^{\perp}\right)\right.
$$

2.2. Où l'on fait apparaître la transformation de Radon de $f$ dans le « $\bar{\partial} »$ de $f_{ \pm} \cdots$

Soit $f$ une fonction $\mathscr{C}^{1}\left(\mathbb{R}^{2}, \mathbb{C}\right)$, on suppose que la transformation de Radon réelle s'annule sur les droites d'un domaine fortement linéairement concave $\Omega$.

Un changement de variable nous permet d'écrire que, pour $z \in \Omega_{ \pm}$:

$$
f_{ \pm}(z)=\int_{s \in \mathbb{R}} \frac{f\left(\Re\left(z_{1}\right)+\vartheta_{1}(z)^{\perp} s, \Re\left(z_{2}\right)+\vartheta_{2}(z)^{\perp} s\right)}{s-i\left\langle\vartheta(z)^{\perp}, \Im(z)\right\rangle} d s
$$

Nous noterons dans la suite $f()$ ou $\partial_{i} f(), i=1,2$, les éléments $f\left(\Re\left(z_{1}\right)+\right.$ $\left.\vartheta_{1}(z)^{\perp} s, \Re\left(z_{2}\right)+\vartheta_{2}(z)^{\perp} s\right)$ ou $\partial_{i} f\left(\Re\left(z_{1}\right)+\vartheta_{1}(z)^{\perp} s, \Re\left(z_{2}\right)+\vartheta_{2}(z)^{\perp} s\right)$.

Proposition 2.1. - Nous avons l'identité suivante

$$
\begin{gathered}
\left(\vartheta_{1}(z) \bar{\partial}_{z_{1}}+\vartheta_{2}(z) \bar{\partial}_{z_{2}}\right) f_{ \pm}(z)= \\
\frac{i}{|\Im(z)|}\left(\vartheta_{1}(z) \frac{\partial}{\Re\left(z_{1}\right)}+\vartheta_{2}(z) \frac{\partial}{\Re\left(z_{2}\right)}\right) \int_{s \in \mathbb{R}} f\left(\Re\left(z_{1}\right)+\vartheta_{1}(z)^{\perp} s, \Re\left(z_{2}\right)+\vartheta_{2}(z)^{\perp} s\right) d s
\end{gathered}
$$


Démonstration. - En effet,

(3)

$$
\left(\vartheta_{1}(z) \bar{\partial}_{z_{1}}+\vartheta_{2}(z) \bar{\partial}_{z_{2}}\right) f_{ \pm}(z)
$$

$$
\begin{aligned}
= & \int_{s \in \mathbb{R}} \frac{\vartheta_{1}(z)\left(1+\bar{\partial}_{z_{1}} \vartheta_{1}(z)^{\perp} s\right) \partial_{1} f()+\vartheta_{1} \bar{\partial}_{z_{1}} \vartheta_{2}(z)^{\perp} s \partial_{2} f()}{s-i\left\langle\vartheta(z)^{\perp}, \Im(z)\right\rangle} d s \\
& +\int_{s \in \mathbb{R}} \frac{\vartheta_{2}(z) \bar{\partial}_{z_{2}} \vartheta_{1}(z)^{\perp} s \partial_{1} f()+\vartheta_{2}(z)\left(1+\bar{\partial}_{z_{2}} \vartheta_{2}(z)^{\perp} s\right) \partial_{2} f()}{s-i\left\langle\vartheta(z)^{\perp}, \Im(z)\right\rangle} d s \\
& +\int_{s \in \mathbb{R}} \frac{i \vartheta_{1}(z)\left(\left\langle\bar{\partial}_{z_{1}} \vartheta(z)^{\perp}, \Im(z)\right\rangle+i \vartheta_{1}(z)^{\perp}\right) f()}{\left(s-i\left\langle\vartheta(z)^{\perp}, \Im(z)\right\rangle\right)^{2}} d s \\
& +\int_{s \in \mathbb{R}} \frac{i \vartheta_{2}(z)\left(\left\langle\bar{\partial}_{z_{2}} \vartheta(z)^{\perp}, \Im(z)\right\rangle+i \vartheta_{2}(z)^{\perp}\right) f()}{\left(s-i\left\langle\vartheta(z)^{\perp}, \Im(z)\right\rangle\right)^{2}} d s
\end{aligned}
$$

On remplace dans les deux premières intégrales la variable $s$ par $s-$ $i\left\langle\vartheta(z)^{\perp}, \Im(z)\right\rangle+i\left\langle\vartheta(z)^{\perp}, \Im(z)\right\rangle$. On obtient alors que :

$$
(4)+(5)=
$$

(8)

$$
\int_{s \in \mathbb{R}}\left(\vartheta_{1}(z) \bar{\partial}_{z_{1}} \vartheta_{1}(z)^{\perp}+\vartheta_{2}(z) \bar{\partial}_{z_{2}} \vartheta_{1}(z)^{\perp}\right) \partial_{1} f()+\left(\vartheta_{1}(z) \bar{\partial}_{z_{1}} \vartheta_{2}(z)^{\perp}+\vartheta_{2}(z) \bar{\partial}_{z_{2}} \vartheta_{2}(z)^{\perp}\right) \partial_{2} f() d s
$$

$$
+\int_{s \in \mathbb{R}} \frac{\left(\vartheta_{1}(z)\left(1+i \bar{\partial}_{z_{1}} \vartheta_{1}(z)^{\perp}\left\langle\vartheta(z)^{\perp}, \Im(z)\right\rangle\right)+i \vartheta_{2}(z) \bar{\partial}_{z_{2}} \vartheta_{1}(z)^{\perp}\left\langle\vartheta(z)^{\perp}, \Im(z)\right\rangle\right) \partial_{1} f()}{s-i\left\langle\vartheta(z)^{\perp}, \Im(z)\right\rangle} d s
$$

$$
+\int_{s \in \mathbb{R}} \frac{\left(i \vartheta_{1}(z) \bar{\partial}_{z_{1}} \vartheta_{2}(z)^{\perp}\left\langle\vartheta(z)^{\perp}, \Im(z)\right\rangle+\vartheta_{2}(z)\left(1+i \bar{\partial}_{z_{2}} \vartheta_{2}(z)^{\perp}\left\langle\vartheta(z)^{\perp}, \Im(z)\right\rangle\right)\right) \partial_{2} f()}{s-i\left\langle\vartheta(z)^{\perp}, \Im(z)\right\rangle} d s .
$$

Par ailleurs, en utilisant le fait que $\left\langle\vartheta(z), \vartheta(z)^{\perp}\right\rangle=0$, et le fait que, puisqu'on a l'égalité, $\Im\left(z_{1}\right) \vartheta_{2}(z)^{\perp}=\Im\left(z_{2}\right) \vartheta_{1}(z)^{\perp}$, on obtient en différentiant,

$$
\left\langle\bar{\partial}_{z_{1}} \vartheta(z)^{\perp}, \Im(z)\right\rangle=\left\langle\bar{\partial}_{z_{2}} \vartheta(z)^{\perp}, \Im(z)\right\rangle=0,
$$

On obtient ainsi que :

$$
(6)+(7)=0
$$

On a aussi,

$$
\vartheta_{1}(z)\left(1+i \bar{\partial}_{z_{1}} \vartheta_{1}(z)^{\perp}\left\langle\vartheta(z)^{\perp}, \Im(z)\right\rangle\right)+i \vartheta_{2}(z) \bar{\partial}_{z_{2}} \vartheta_{1}(z)^{\perp}\left\langle\vartheta(z)^{\perp}, \Im(z)\right\rangle=0 .
$$

En effet, par un calcul élémentaire, le terme ci-dessus, en remplaçant $\left(\vartheta_{1}(z)^{\perp}, \vartheta_{2}(z)^{\perp}\right)=\left(-\vartheta_{2}(z), \vartheta_{1}(z)\right)$ par $\left(\frac{\Im\left(z_{1}\right)}{|\Im(z)|}, \frac{\Im\left(z_{2}\right)}{|\Im(z)|}\right)$, s'annule bien.

Ainsi dans la somme $(9)+(10)$, le facteur de $\partial_{1} f$ s'annule et de la même manière celui de $\partial_{2} f$. 
On peut donc conclure que :

$$
\left(\vartheta_{1}(z) \bar{\partial}_{z_{1}}+\vartheta_{2}(z) \bar{\partial}_{z_{2}}\right) f_{ \pm}(z)=
$$

$\int_{s \in \mathbb{R}}\left(\vartheta_{1}(z) \bar{\partial}_{z_{1}} \vartheta_{1}(z)^{\perp}+\vartheta_{2}(z) \bar{\partial}_{z_{2}} \vartheta_{1}(z)^{\perp}\right) \partial_{1} f()+\left(\vartheta_{1}(z) \bar{\partial}_{z_{1}} \vartheta_{2}(z)^{\perp}+\vartheta_{2}(z) \bar{\partial}_{z_{2}} \vartheta_{2}(z)^{\perp}\right) \partial_{2} f() d s$

Or, on déduit des relations $\Im\left(z_{1}\right) \vartheta_{2}(z)^{\perp}=\Im\left(z_{2}\right) \vartheta_{1}(z)^{\perp}$ et $\left(\vartheta_{1}(z)^{\perp}\right)^{2}+$ $\left(\vartheta_{2}(z)^{\perp}\right)^{2}=1$, que

$$
\vartheta_{1}(z) \bar{\partial}_{z_{1}} \vartheta_{1}(z)^{\perp}+\vartheta_{2}(z) \bar{\partial}_{z_{2}} \vartheta_{1}(z)^{\perp}=i \frac{\vartheta_{2}(z)^{\perp}}{|\Im(z)|}=i \frac{\vartheta_{1}(z)}{|\Im(z)|}
$$

De même,

$$
\vartheta_{1}(z) \bar{\partial}_{z_{1}} \vartheta_{2}(z)^{\perp}+\vartheta_{2}(z) \bar{\partial}_{z_{2}} \vartheta_{2}(z)^{\perp}=-i \frac{\vartheta_{1}(z)^{\perp}}{|\Im(z)|}=i \frac{\vartheta_{2}(z)}{|\Im(z)|}
$$

où l'on a noté par $|\Im(z)|,\left(\Im\left(z_{1}\right)^{2}+\Im\left(z_{2}\right)^{2}\right)^{\frac{1}{2}}$

On en déduit que :

$$
(11)=\frac{i}{\Im(z)} \int_{s \in \mathbb{R}} \vartheta_{1}(z) \partial_{1} f()+\vartheta_{2}(z) \partial_{2} f() d s
$$

Or ce dernier terme n'est rien d'autre que :

$\frac{i}{|\Im(z)|}\left(\vartheta_{1}(z) \frac{\partial}{\Re\left(z_{1}\right)}+\vartheta_{2}(z) \frac{\partial}{\Re\left(z_{2}\right)}\right) \int_{s \in \mathbb{R}} f\left(\Re\left(z_{1}\right)+\vartheta_{1}(z)^{\perp} s, \Re\left(z_{2}\right)+\vartheta_{2}(z)^{\perp} s\right) d s$

La transformation de Radon s'annulant, ce dernier terme s'annule. On en déduit que :

$$
\left(\vartheta_{1}(z) \bar{\partial}_{z_{1}}+\vartheta_{2}(z) \bar{\partial}_{z_{2}}\right) f_{ \pm}(z)=0
$$

À présent, si l'on reprend la formule de Plemelj -Sohotsky (1), on voit que $h$ se décompose en la différence de deux fonctions respectivement holomorphes sur le demi-plan des parties imaginaires positives (resp. négatives) de la « droite complexe » $\mathbb{C}$, (on remplace simplement $x$ par $z$ ).

L'équation (2) montre que $f$ se décompose sur la droite complexe $\langle\vartheta(x), \xi-$ $x\rangle=0$, en la différence de deux fonctions holomorphes relativement à la variable complexe $Z=\langle\vartheta(x), z\rangle$. De même, pour $f_{ \pm}$, on exprime cette dernière fonction selon une représentation de Cauchy pour la variable complexe $Z=\langle\vartheta(z), z\rangle$. Si bien que l'on peut immédiatement conclure que $\left(-\vartheta_{2}(z) \bar{\partial}_{z_{1}}+\vartheta_{1}(z) \bar{\partial}_{z_{2}}\right) f_{ \pm}(z)$ s'annule.

On en déduit donc que $\bar{\partial} f_{ \pm}=0$ sur respectivement $\Omega_{ \pm}$.

Nous sommes maintenant en mesure d'établir le théorème : 
Démonstration du théorème 1.4. - Nous venons de déduire de l'annulation de la transformation de Radon de $f$ que, $\bar{\partial} f_{ \pm}=0$.

De plus, $f_{ \pm}$vérifie une estimation en $\Theta\left(\frac{1}{|z|^{2}}\right)$. En effet, en développant l'expression, on a :

$\left.f_{ \pm}(z)=\int_{\langle\vartheta(z), \xi-z\rangle=0, \Im(\xi)=\left(-\vartheta_{2}(z), \vartheta_{1}(z)\right)} \frac{f(\Re(\xi)) \cdot \operatorname{det}(\Im(\xi), \vartheta(z))}{-\vartheta_{2}(z)\left(\Re\left(\xi_{1}\right)-z_{1}\right)+\vartheta_{1}(z)\left(\Re\left(\xi_{2}\right)-z_{2}\right)} \vartheta(z) d \xi\right\rfloor \omega(\xi)$.

On développe en série dans l'expression ci-dessus le noyau

$$
\frac{1}{-\vartheta_{2}(z)\left(\Re\left(\xi_{1}\right)-z_{1}\right)+\vartheta_{1}(z)\left(\Re\left(\xi_{2}\right)-z_{2}\right)} .
$$

On pose $X:=-\vartheta_{2}(z) \Re\left(\xi_{1}\right)+\vartheta_{1}(z) \Re\left(\xi_{2}\right), Z:=\vartheta_{2}(z) z_{1}-\vartheta_{1}(z) z_{2}$. Et le terme en $\frac{1}{Z}$, n'est rien d'autre que

$$
\left.\int_{\langle\vartheta(z), \xi-z\rangle=0, \Im(\xi)=\left(-\vartheta_{2}(z), \vartheta_{1}(z)\right)} f(\Re(\xi)) \vartheta(z) d \xi\right\rfloor \omega(\xi) \cdot \operatorname{det}\left(\vartheta^{\perp}(z), \vartheta(z)\right) .
$$

Ce terme s'annule puis que ce n'est rien d'autre que la transformation de Radon de $f$ sur la droite $\Re(\langle\vartheta(z), \xi-z\rangle)=0$. Le développement de $f_{ \pm}$, est donc bien en $\theta\left(\frac{1}{|z|^{2}}\right)$.

Remarque. - Pour une application $f$ appartenant à $\mathscr{C}^{2}\left(\mathbb{R}^{2}, \mathbb{C}\right)$, intégrable le long des droites de $\mathbb{R}^{2}$, on a, pour tout $x$ de $\mathbb{R}^{2}$ :

$$
\begin{aligned}
f(x) & =\lim _{\varepsilon \longrightarrow 0} \frac{1}{(2 \pi i)^{2}} \int_{y \in \mathbb{R}^{2}} \int_{\theta \in S^{1}} f(y) \frac{\omega^{\prime}(\theta) \wedge \omega(y)}{(\langle\theta, y-x\rangle+i \epsilon)^{2}} \\
& =\lim _{\varepsilon \longrightarrow 0} \frac{1}{(2 \pi i)^{2}} \int_{t \in \mathbb{R}} \int_{\theta \in S^{1}} \frac{(R f)_{\mathbb{R}}(\theta, t)}{(t-\langle\theta, x\rangle+i \epsilon)^{2}} \omega^{\prime}(\theta) \wedge d t
\end{aligned}
$$

Pour $x=\left(x_{1}, x_{2}\right) \in \Omega$, on note $\Omega_{x}^{*}=\left\{(\theta, t) \in \Omega^{*} \mid\left\{y \in \mathbb{R}^{2} \mid t+\theta_{1} y_{1}+\theta_{2} y_{2}=0\right\} \subset\right.$ $\left.\Omega, t+\theta_{1} x_{1}+\theta_{2} x_{2}=0\right\}$. Supposons maintenant que cette application génère une transformation de Radon réelle s'annulant dans $\Omega^{*}$, domaine dual de $\Omega$. Supposons la projection $\pi\left(\Omega^{*}\right)$ de $\Omega^{*}$ sur $S^{1}$ constituée de deux composantes connexes (et à transformation linéaire près, une des deux composantes connexes de $\pi\left(\Omega^{*}\right)^{\perp}$ sera contenue dans $S_{+}^{1}$ et l'autre dans $S_{-}^{1}, S_{ \pm}^{1}=\left\{\theta=\left(\theta_{1}, \theta_{2}\right) \in\right.$ $\left.\left.S^{1} \mid \pm \theta_{2}>0\right\}\right)$. Soit la décomposition en deux composantes connexes $A_{ \pm}$ de $S^{1} \backslash \pi\left(\Omega^{*}\right)$. Et notons $B_{ \pm}$(resp. $\left.B_{x, \pm}\right)$, les deux composantes connexes de $\pi\left(\Omega^{*}\right)\left(\right.$ resp. $\left.\pi\left(\Omega_{x}^{*}\right)\right)$, choisies telles que $B_{ \pm}^{\perp} \subset S_{ \pm}^{1}$ (resp. $\left.B_{x, \pm}^{\perp} \subset S_{ \pm}^{1}\right)$. Pour $\theta \in$ $\pi\left(\Omega^{*}\right)$, notons $a_{\theta}$ et $b_{\theta}$ les réels tels que $] a_{\theta}, b_{\theta}\left[=\left\{t \in \mathbb{R} \mid(\theta, t) \in \Omega^{*}\right\}\right.$. Alors, de 
la formule précédente on déduit, puisque $(R f)_{\mathbb{R}}(\theta, t)=0$ pour $(\theta, t) \in \Omega^{*}$ :

$$
\begin{aligned}
f(x)= & \lim _{\varepsilon \longrightarrow 0}\left(\frac{1}{(2 \pi i)^{2}} \int_{\theta \in A_{+}} \int_{t \in \mathbb{R}} \frac{(R f)_{\mathbb{R}}(\theta, t)}{(t-\langle\theta, x\rangle+i \epsilon)^{2}} \omega^{\prime}(\theta) \wedge d t\right. \\
& +\frac{1}{(2 \pi i)^{2}} \int_{\theta \in B_{+}} \int_{t \in \mathbb{R} \backslash] a_{\theta}, b_{\theta}[} \frac{(R f)_{\mathbb{R}}(\theta, t)}{(t-\langle\theta, x\rangle+i \epsilon)^{2}} \omega^{\prime}(\theta) \wedge d t \\
& +\frac{1}{(2 \pi i)^{2}} \int_{\theta \in A_{-}} \int_{t \in \mathbb{R}} \frac{(R f)_{\mathbb{R}}(\theta, t)}{(t-\langle\theta, x\rangle+i \epsilon)^{2}} \omega^{\prime}(\theta) \wedge d t \\
& +\frac{1}{(2 \pi i)^{2}} \int_{\theta \in B_{-}} \int_{t \in \mathbb{R} \backslash] a_{\theta}, b_{\theta}[} \frac{(R f)_{\mathbb{R}}(\theta, t)}{(t-\langle\theta, x\rangle+i \epsilon)^{2}} \omega^{\prime}(\theta) \wedge d t .
\end{aligned}
$$

Or, l'application qui à $z$ associe $\frac{1}{(t-\langle\theta, z\rangle+i \epsilon)}, \varepsilon>0, t \in \mathbb{R}, \theta \in S^{1}$, est holomorphe sur $\left\{z \in \mathbb{C}^{2} \mid\langle\theta, \Im(z)\rangle<\varepsilon\right\}$. Ainsi, $\theta$ parcourant $S^{1}$, compte tenu de ce que, pour $z \in \mathbb{C}^{2}$ tel que $x=\Re(z) \in \Omega$ et pour $\theta \in B_{x, \pm}$, $(t-\langle\theta, z\rangle+i \epsilon)$ n'est jamais nul, chacun des deux premiers termes de $f$ est respectivement holomorphes sur $\cap_{\theta \in\left(A_{+} \cup\left(B_{+} \backslash B_{x,+}\right)\right.}\left\{z \in \mathbb{C}^{2} \mid\langle\theta, \Im(z)\rangle<0\right\}$ et $\cap_{\theta \in\left(A_{-} \cup\left(B_{-} \backslash B_{x,-}\right)\right)}\left\{z \in \mathbb{C}^{2} \mid\langle\theta, \Im(z\rangle)<0\right\}$.

Nous avons obtenu précédemment une décomposition de $f$ en résidus de deux fonctions holomorphes sur $\Omega_{+}$resp. sur $\Omega_{-}$. Or ces deux derniers ensembles sont des réunions de cônes variant avec $x \in \Omega$. Ici, nous obtenons une décomposition plus rigide puisque $\cap_{\theta \in\left(A_{ \pm} \cup\left(B_{ \pm} \backslash B_{x, \pm}\right)\right.}\left\{z \in \mathbb{C}^{2} \mid\langle\theta, \Im(z)\rangle<0\right\}$ peut être vide lorsque $x$ se rapproche du bord de $\Omega$.

En revanche, si l'on se fixe un compact dans $\Omega$, on peut décomposer $f$ par la formule d'inversion de Radon globale, selon le même procédé établi plus haut, pour que $f$ se décompose en différence de deux fonctions holomorphes sur des hémi-cônes $\widetilde{\Omega}_{ \pm}$strictement inclus dans $\Omega_{ \pm}$. La décomposition existe bien mais elle n'est pas optimale.

Le résultat qui suit, connu sous le nom de théorème de support, que l'on retrouve démontré chez John [9] puis généralisé par Cormack [2] et Helgason [6].

Corollaire 2.2. - Soit $f$ une fonction de $\mathscr{C}^{2}\left(\mathbb{R}^{2}, \mathbb{C}\right)$ à support compact $K$ dans $\mathbb{R}^{2}$. On suppose que la transformation de Radon réelle s'annule dans un domaine linéairement concave $\Omega$.On suppose qu'il existe une droite $D$ de $\mathbb{R}^{2}$ entièrement contenue dans $\Omega$ telle que $D \cap K=\varnothing$. Alors $f$ s'annule dans $\Omega$.

Démonstration. - $f$ se décompose en la différence de deux fonctions respectivement holomorphe dans les domaines $\Omega_{+}$et $\Omega_{-}$. On sait que les fonctions $f_{+}$ et $f_{-}$coïncident sur $\Omega \backslash K$.

En posant $F=f_{+}$sur $\Omega_{+}$et $F=f_{-}$sur $\Omega_{-}$, on obtient une fonction prolongée, définie sur $\Omega_{+} \cup \Omega_{-} \cup(\Omega \backslash K)$. 
On a supposé qu'il existait une droite $D$ de $\mathbb{R}^{2}$ entièrement contenue dans $\Omega$ telle que $D \cap K=\varnothing$. Il existe alors deux ouverts connexes, $\Omega_{1}$ et $\Omega_{2}$, tels que $\Omega_{1} \cup D \cup \Omega_{2}=\Omega$, et tels que les éléments $\Omega_{1}, D, \Omega_{2}$, soient deux à deux disjoints. De plus, il existe une droite $D^{\prime}$, parallèle à $D$, par exemple contenue dans $\Omega_{2}$, de paramètre $(\theta, t) \in S^{1} \times \mathbb{R}$, telle que, si $A$ désigne l'ensemble $\{\alpha \in \mathbb{R} \mid(\theta, \alpha) \in$ $\left.\Omega^{*},\left\{\left(x_{1}, x_{2}\right) \in \mathbb{R}^{2} \mid \alpha+\theta_{1} x_{1}+\theta_{2} x_{2}=0\right\} \cap K=\varnothing\right\}, t \in\{\inf (A), \sup (A)\}$. (Il est possible que les droites de paramètres $(\theta, \inf (A))$ et $(\theta, \sup (A))$ n'appartiennent pas à l'ouvert $\Omega^{*}$, ceci signifie alors que toutes les droites de $\Omega^{*}$ parallèles à $D$ ont une intersection vide avec $K$, il faut alors choisir localement autour de la direction de $D$, une autre droite d'intersection vide avec $K$, et reprendre la construction ci-dessus jusqu'à ce qu'une des droites de paramètres $(\theta, \inf (A))$ ou $(\theta, \sup (A))$ appartiennent à $\Omega^{*}$. Bien entendu, si ceci n'est possible pour aucune des droites de $\Omega$, alors on conclut que $f$ est identiquement nulle sur $\Omega$ ).

On considère un voisinage $V\left(D^{\prime}\right)$ de droites autour de $D^{\prime}$, incluses dans $\Omega^{*}$ et parallèles à $D^{\prime}$. Nous sommes maintenant en mesure d'appliquer à $F$ définie sur $\cup_{x \in V\left(D^{\prime}\right) \backslash\left(V\left(D^{\prime}\right) \cap K\right)} \Omega_{x, \pm}$, avec $\Omega_{x, \pm}=\{x\} \cup\left\{z \in \Omega_{ \pm} \mid \Re(z)=x\right\}$. Cet ensemble contenant $V\left(D^{\prime}\right) \backslash\left(V\left(D^{\prime}\right) \cap K\right) \subset \Omega$, appliquons le théorème d'edge-of-thewedge sur cet ensemble sur lequel notre fonction $F$ est définie, holomorphe sur ses parties de partie imaginaire respectivement strictement positive et négative. Par le théorème d'edge-of-the-wedge, on la prolonge localement holomorphiquement autour de $V\left(D^{\prime}\right) \backslash\left(V\left(D^{\prime}\right) \cap K\right)$ sur un ensemble $\Delta$. En complexifiant les droites de $V\left(D^{\prime}\right)$, on dispose maintenant d'une famille de droites complexes parallèles, qui nous place, dans $\mathbb{C}^{2}$, dans les conditions d'application du principe de continuité d'Hartog's ou Kontinuitatsatz. Si bien que, sur $V\left(D^{\prime}\right)$, les fonctions $f_{+}$et $f_{-}$coïncident. De proche en proche, on déduit que $F$ se prolonge à $\Omega$, (ce processus est possible puisque le support de $f$ est compact). Finalement $f$ s'annule sur $\Omega$.

Remarque. - Ce résultat est bien celui démontré par John [9] puis généralisé par Cormack [2] et Helgason [6]. En effet, il suffit de prendre la droite $D$ mentionnée dans le corollaire 2.2., c'-à-d incluse strictement dans $\Omega$ et d'intersection nulle avec le support de $f$, et la considérer comme droite à l'infini dans $\mathbb{R P}^{2}$. On se trouve alors dans les conditions du théorème démontré par les auteurs ci-dessus.

Remarque. - Si $f$ est une fonction à support compact dans un domaine linéairement concave $\Omega$ de $\mathbb{R}^{2}$, et si l'on suppose que sa transformation de Radon réelle s'annule sur les droites de $\Omega$, alors $f$ s'annule. 
Remarque. - Le résultat qui suit est une application de raffinements du théorème d'《edge-of-the-wedge », que l'on doit à Kolm et Nagel [10]. Mais, c'est à Boman dans [1], que l'on doit la formulation de la proposition qui suit, ici démontrée à la lumière du théorème 1.4. du présent article.

Il faut ici signaler que Boman, dans [1], expose une démonstration qui utilise essentiellement un théorème de régularité micro-locale des solutions de l'équation $R(f)_{\mathbb{R}}=0$.

Proposition 2.3. - Soit un $K$ un compact convexe propre de $\mathbb{R}^{2}$, et $f$ une fonction de $\mathscr{C}^{2,2}\left(\mathbb{R}^{2}\right)$. Supposons qu'il existe un segment $I$ de $\mathbb{R}^{2}$, borné, tel que la droite $D$ qu'il génère appartienne à $\left(\mathbb{R}^{2} \backslash K\right)^{*}$. Supposons de plus que la transformation de Radon de $f$ soit nulle selon toutes les droites de $\left(\mathbb{R}^{2} \backslash K\right)^{*}$ et que toutes les dérivées, à tous les ordres de $f$ sur I s'annulent. Alors, $f$ s'annule au voisinage de $I$.

Démonstration. - Soit a,b les éléments du bord de $I$. Il existe, aux points $a$ et $b$, des voisinages $V$ et $V^{\prime}$, (toujours paramétrés par $S^{1} \times \mathbb{R}$ ), de la droite $D$, dont tous les éléments passent respectivement par $a$ et $b$. Notons $C=\pi_{1}(V) \cap \pi_{1}\left(V^{\prime}\right)$, où $\pi_{1}$ désigne toujours la première projection sur $S^{1}$. Cette intersection est non vide. (En effet,l'application qui à un point $x$ de $I$ associe l'ouvert $\pi_{1}\left(V_{x}\right)$ des directions des droites de $K^{*}$ passant par ce point est continue, il existe donc un voisinage $U_{x}$ de $x$ dans $I$ tel que $\cap_{x \in U_{x}} \pi_{1}\left(V_{x}\right) \neq \varnothing$ et en extrayant un recouvrement fini du compact $I$, à partir des $U_{x}$, on déduit que le cône $C$ de direction de droites autour de $D$ est non vide). Soit $\mathscr{C} \subset \mathbb{R}^{2}$ le cône $\mathscr{C}=\bigcup_{ \pm} \mathscr{C}_{ \pm}=\left\{x \in \mathbb{R}^{2} \mid \exists \lambda \in \mathbb{R}_{ \pm}^{*}, c \in C, x=\lambda c\right\}$.

Nous sommes alors en mesure d'appliquer un raffinement du théorème d'« edge-of-the-wedge », (Kolm A., Nagel B. [10]),qui stipule qu'étant donné une courbe analytique $J$, parametrée par $x(t), t \in \mathbb{R}$, un cône $\mathscr{C}=\bigcup_{ \pm} \mathscr{C}_{ \pm}$, tels que $x^{\prime}(t) \in \mathscr{C}$, pour tout $t$, et deux fonctions respectivement holomorphes sur $J+i \mathscr{C}_{ \pm}$telles que toutes leurs dérivées, à tous les ordres coïncident sur $J$, alors il existe un prolongement analytique commun aux deux fonctions, holomorphe sur un voisinage complexe de $J$. Dans notre cas, la transformation de Radon de $f$ s'annulant sur $\left(\mathbb{R}^{2} \backslash K\right)^{*}, f$ se décompose en la différence de deux fonctions holomorphes $f=f_{+}-f_{-}$, et les dérivées de $f$ s'annulant à tous les ordres sur $I$, les dérivées de $f_{+}$et de $f_{-}$coïncident sur $I$. Nous sommes alors dans les conditions d'application du théorème d' « edge-of-the-wedge » généralisé, démontré dans [10].

Remarque. - Cette proposition peut être encore améliorée. Avec les mêmes hypothèses qu'en 2.3., on peut conclure que $f$ s'annule sur l'enveloppe C-convexe $C(I)$ du segment $I$, grâce à une amélioration du théorème d'edge-of-the-wedge due à Vladimirov [13]. On définit $C(I)$ comme étant le plus petit ouvert qui 
contient $I$ et qui a la propriété que, si $x^{\prime}$ et $x^{\prime \prime}$ peuvent être joints par une 《 courbe C-similaire » (cf. définition ci-après) entièrement contenue dans $C(I)$, alors toutes les courbes C-similaire joignant $x^{\prime}$ et $x^{\prime \prime}$ sont contenues dans $C(I)$. Une courbe de classe $\mathscr{C}^{1}$ est dite C-similaire, pour un cône $C$, si, en chaque point de la courbe, la direction du vecteur tangent est contenue dans $C$.

Géométriquement, l'enveloppe C-convexe de $I$ correspond à un parallélogramme déterminé par les directions limites de $\Omega_{x}^{*}$, ( $\Omega$ est ici le complémentaire de $K$ ), pour $x$ appartenant aux extrémités du segment $I$.

\section{La caractérisation de l'image de la transformation de Radon réelle}

Pour caractériser complètement la transformation de Radon réelle, nous avons le

Proposition 3.1. - Soit $\Omega$ un domaine fortement linéairement concave de $\mathbb{R}^{2}$. Soit $g \in \mathscr{C}^{3,2}\left(\bar{\Omega}^{*}, \mathbb{C}\right)$ telle que $g(\theta, t)=g(-\theta,-t)$, alors il existe un prolongement $\widetilde{g} \in \mathscr{C}^{3,2}\left(\mathbb{R}^{2, *}, \mathbb{C}\right)$ de g tel que $\widetilde{g}(\theta, t)=\widetilde{g}(-\theta,-t)$.

Démonstration. - On revient aux notations ci-dessus.

Soit $\pi$ la première projection de $\Omega^{*}$ dans $S^{1} \times \mathbb{R}$. Nous choisirons de pointer le repère de $\mathbb{R}^{2}$ en un élément de $\Omega$. Supposons que le complémentaire de $\Omega$ est constitué de deux composantes connexes. Le bord de $\pi\left(\Omega^{*}\right)$ est alors constitué de quatre éléments que nous noterons $\pm \theta_{1}$ et $\pm \theta_{2}$. L'ensemble $S^{1} \backslash \pi\left(\Omega^{*}\right)$ est composé de deux composantes connexes, l'une $A$ de bord les éléments $\theta_{1}$ et $-\theta_{2}$ et $B$ de bord les éléments $-\theta_{1}$ et $\theta_{2}$.

$g$ est connue pour tout $(\theta, t) \in \pi\left(\Omega^{*}\right) \times\left[a_{\theta}, b_{\theta}\right]$, où $a_{\theta}$ et $b_{\theta}$ sont des réels dépendant de $\theta$. Nous allons prolonger successivement $g$ sur l'ensemble des $\left.(\theta, t) \in \pi\left(\Omega^{*}\right) \times(]-\infty, a_{\theta}\right] \cup\left[b_{\theta}, \infty[)\right.$ puis sur l'ensemble des $(\theta, t) \in A \times[0, \infty[$ et sur l'ensemble des $(\theta, t) \in B \times]-\infty, 0]$. Nous compléterons enfin par parité. Nous noterons $\widetilde{g}$ ce prolongement.

Notons $c$ le réel $2 \max _{\theta \in \pi\left(\Omega^{*}\right)}\left(\left|a_{\theta}\right|,\left|b_{\theta}\right|\right)$. Soit le polynôme interpolateur de degré 8 , tel que, pour des réels $a, b, \alpha_{i}, \beta_{i}, i=0,1,2,3, P^{(i)}(a)=\alpha_{i}, P^{(i)}(b)=\beta_{i}$. Nous le noterons $P_{a, b ; \alpha_{i}, \beta_{i}}$. Ces polynômes sont choisis pour que le prolongement construit ci-dessous soit $\mathscr{C}^{3}$.

On pose alors, pour $b_{\theta} \leq t \leq c$,

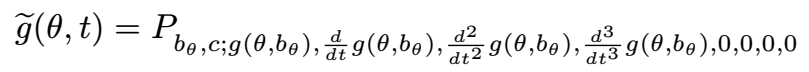

et pour $t \geq c, \widetilde{g}(\theta, t)=0$. Pour $-c \leq t \leq a_{\theta}$,

$$
\widetilde{g}(\theta, t)=P_{a_{\theta},-c ; g\left(\theta, a_{\theta}\right), \frac{d}{d t} g\left(\theta, a_{\theta}\right), \frac{d^{2}}{d t^{2}} g\left(\theta, a_{\theta}\right), \frac{d^{3}}{d t^{3}} g\left(\theta, a_{\theta}\right), 0,0,0,0}
$$

et $\widetilde{g}(\theta, t)=0$ pour $t \leq-c$. 
Complétons alors, pour $t \in \mathbb{R}$ et $\theta \in A, h(\theta, t)=P_{t} \circ \arccos (\theta)$ où

$$
P_{t}=P_{\arccos \left(\theta_{1}\right), \arccos \left(-\theta_{2}\right) ; G\left(\theta_{1}, t\right), G\left(-\theta_{2}, t\right)} .
$$

où l'on a noté $G\left(\theta_{1}, t\right)$, resp. $G\left(-\theta_{2}, t\right)$, le triplet $\left(g\left(\theta_{1}, t\right), \frac{d}{d \theta} g\left(\theta_{1}, t\right), \frac{d^{2}}{d \theta^{2}} g\left(\theta_{1}, t\right)\right.$, $\left.\frac{d^{3}}{d \theta^{3}} g\left(\theta_{1}, t\right)\right)$, resp. $\left(g\left(-\theta_{2}, t\right), \frac{d}{d \theta} g\left(-\theta_{2}, t\right), \frac{d^{2}}{d \theta^{2}} g\left(-\theta_{2}, t\right), \frac{d^{3}}{d \theta^{3}} g\left(-\theta_{2}, t\right)\right)$.

On procède de même pour $\theta \in B$. Bien entendu, le prolongement ci-dessus appartient bien à $\mathscr{C}^{3,2}$ puisqu'il s'annule pour tout $(\theta, t)$, avec $t$ suffisamment grand.

Si le complémentaire de $\Omega$ est constitué d'une seule composante connexe. Alors, il faut distinguer le cas où celle-ci est compacte et l'autre cas où celle-ci n'est pas borné. Dans le second cas le prolongement est le même que ci-dessus, à l'exception que pour $a_{\theta}=-\infty$ ou $b_{\theta}=+\infty$, il est inutile de prolonger $g(\theta, t)$ pour $t<a_{\theta}$ (resp. $t>b_{\theta}$ ). Le prolongement est encore $\mathscr{C}^{3,2}$ puisque, pour tout $t$, la norme du polynôme interpolateur $P_{t}$ est majorée par une combinaison polynômiale des dérivées partielles jusqu'à l'ordre 3 de $g\left(\theta_{1}, t\right)$ et $g\left(-\theta_{2}, t\right)$; la résultante suivra bien une évolution en $\theta\left(\frac{1}{t^{2}}\right)$.

Enfin, dans le cas où le complémentaire de $\Omega$ est compact, il s'agit alors de prolonger $g$ pour les couples $(\theta, t) \in S^{1} \times\left[a_{\theta}, b_{\theta}\right]$. On note alors $c=$ $\frac{1}{2} \min _{\theta \in S^{1}}\left(a_{\theta}, b_{\theta}\right)$. Et on pose, pour $c \leq t \leq b_{\theta}$,

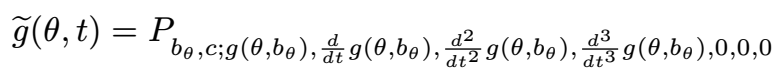

et nulle pour $0 \leq t \leq c$ et pour $a_{\theta} \leq t \leq-c_{\theta}$,

$$
\widetilde{g}(\theta, t)=P_{a_{\theta},-c ; g\left(\theta, a_{\theta}\right), \frac{d}{d t} g\left(\theta, a_{\theta}\right), \frac{d^{2}}{d t^{2}} g\left(\theta, a_{\theta}\right), \frac{d^{3}}{d t^{3}} g\left(\theta, a_{\theta}\right), 0,0,0}
$$

et $\widetilde{g}$ nulle pour $-c_{\theta} \leq t \leq 0$.

Démonstration du théorème 1.5. - On rappelle un théorème établi par Radon [12] : Si $g$ est une fonction de $\mathscr{C}^{3,2}\left(S^{1} \times \mathbb{R}, \mathbb{C}\right),\left(\right.$ c'-à-d $\mathscr{C}^{3}\left(S^{1} \times \mathbb{R}, \mathbb{C}\right)$, et vérifiant, uniformément par rapport à $\theta \in S^{1}$, pour $i+j=0,1,2,3, \frac{d^{i}}{d \theta^{i}} \frac{d^{j}}{d t^{j}} g(\theta, t)=$ $\Theta\left(\frac{1}{t^{2}}\right)$ quand $t$ tend vers l'infini), telle que $g(\theta, t)=g(-\theta,-t)$, alors il existe $f \in \mathscr{C}^{1}\left(\mathbb{R}^{2}, \mathbb{C}\right)$, intégrable sur les droites de $\mathbb{R}^{2}$, telle que $(R f)_{\mathbb{R}}=g$.

Soit $g$ tel qu'il nous est donné par les hypothèses du théorème 1.5., alors on prolonge $g$ par $\widetilde{g}$ conformément à la proposition 3.1., puis on applique maintenant à $\widetilde{g}$ le théorème de Radon [12], pour obtenir $f$ dans $\mathscr{C}^{1}\left(\mathbb{R}^{2}, \mathbb{C}\right)$, intégrable sur toutes les droites de $\Omega^{*}$, telle que la transformée de Radon de $f$ sur $\Omega^{*}$ est $g$.

Nous sommes en mesure d'établir la : 
Démonstration du théorème 1.6. - À présent, $f$ est une fonction appartenant à $\mathscr{C}_{c}^{3}\left(\mathbb{R}^{2}, \mathbb{C}\right)$. D'après la proposition 3.1., il existe un prolongement de $R(f)_{\mathbb{R}}$, noté $\widehat{R(f)_{\mathbb{R}}}$, défini sur $S^{1} \times \mathbb{R}$. Par le théorème de Radon [12], il existe une fonction $\mathrm{G}$, notée $B\left(R(f)_{\mathbb{R}}\right), \mathscr{C}^{1}$, telle que $R(G)_{\mathbb{R}}=\overline{R(f)_{\mathbb{R}}}$. Appliquons à $B\left(R(f)_{\mathbb{R}}\right)$ la formule d'inversion de Radon, on a :

$$
B\left(R(f)_{\mathbb{R}}\right)(x)=\lim _{\varepsilon \rightarrow 0} \frac{1}{(2 \pi i)^{2}} \int_{t \in \mathbb{R}} \int_{\theta \in S^{1}} \frac{\overline{R(f)_{\mathbb{R}}}(\theta, t)}{(t-\langle\theta, x\rangle+i \varepsilon)^{2}} \omega^{\prime}(\theta) \wedge d t
$$

Les transformées de Radon de $f$ et de $B\left(R(f)_{\mathbb{R}}\right)$ coïncident sur $\Omega^{*}$, soit, $R\left(B\left(R(f)_{\mathbb{R}}\right)\right)_{\mathbb{R}}((\theta, t))=R(f)_{\mathbb{R}}(\theta, t)$, pour tout $(\theta, t)$ de $\Omega^{*}$. Nous sommes en mesure d'appliquer le théorème 1.4. :

$$
f-B\left(R(f)_{\mathbb{R}}\right)=\left(f-B\left(R(f)_{\mathbb{R}}\right)\right)_{+}+\left(f-B\left(R(f)_{\mathbb{R}}\right)\right)_{-},
$$

avec, pour $z \in \Omega_{ \pm}$:

$$
\begin{aligned}
\left.\left(f-B(R(f))_{\mathbb{R}}\right)\right)_{ \pm}(z)= & \mp \frac{1}{2 \pi i} \int_{\langle\vartheta(z), \xi-z\rangle=0, \Im(\xi)=\vartheta(z)^{\perp}}\left(f(\Re(\xi))-B\left(R(f)_{\mathbb{R}}\right)\right. \\
& \left.\left.(\Re(\xi))) \operatorname{det}\left(\vartheta(z), \frac{\Im(\xi)}{\langle\Im(\xi), \Re(\xi)-z\rangle}\right)(\vartheta(z) d \xi)\right\rfloor \omega(\xi)\right) .
\end{aligned}
$$

On note alors $K_{ \pm} f=\left(f-B\left(R(f)_{\mathbb{R}}\right)\right)_{ \pm}$. Les transformations de Radon de $f$ et de $B\left(R(f)_{\mathbb{R}}\right)$ étant identique, on en déduit que $R\left(K_{+} f+K_{-} f\right)_{\mathbb{R}}$ s'annule.

En écrivant $f=f-B\left(R(f)_{\mathbb{R}}\right)+B\left(R(f)_{\mathbb{R}}\right)$, on obtient le théorème.

\section{BIBLIOGRAPHIE}

[1] J. BOMAN - «Holmgren's uniqueness theorem and support theorems for real analytic Radon transforms », in Geometric analysis (Philadelphia, PA, 1991), Contemp. Math., vol. 140, Amer. Math. Soc., 1992, p. 23-30.

[2] A. M. CoRmack - « Representation of a function by its line integrals, with some radiological applications », J. Appl. Phys. 34 (1963), p. 27222727.

[3] P. FUNK - « Über eine geometrische Anwendung der abelschen Integralgleichung », Math. Ann. 77 (1915), p. 129-135.

[4] I. M. Gel'FAnd, M. I. Graev \& N. Y. Vilenkin - Generalized functions. Vol. 5 : Integral geometry and representation theory, Academic Press, 1966.

[5] S. G. Gindikin \& G. M. Henkin - «Integral geometry for $\bar{\partial}$-cohomology in $q$-linearly concave domains in $\mathrm{CP}^{n} »$, Funktsional. Anal. i Prilozhen. 12 (1978), p. 6-23. 
[6] S. HeLGASON - «A duality in integral geometry; some generalizations of the Radon transform », Bull. Amer. Math. Soc. 70 (1964), p. 435-446.

[7] G. M. Henkin - «Abel-Radon transform and applications », in The legacy of Niels Henrik Abel, Springer, 2004, p. 567-584.

[8] G. M. Henkin \& P. L. Polyakov - « Residue integral formulas and the Radon transform for differential forms on $q$-linearly concave domains », Math. Ann. 286 (1990), p. 225-254.

[9] F. JoHN - «Abhängigkeiten zwischen den Flächenintegralen einer stetigen Funktion », Math. Ann. 111 (1935), p. 541-559.

[10] A. Kolm \& B. NAGel - «A generalized edge of the wedge theorem », Comm. Math. Phys. 8 (1968), p. 185-203.

[11] A. Martineau - «Equations différentielles d'ordre infini », Bull. Soc. Math. France 95 (1967), p. 109-154.

[12] J. RADON - « Über die Bestimmung von Funktionen durch ihre Integralwerte längs gewisser Mannigfaltigkeiten », in Computed tomography (Cincinnati, Ohio, 1982), Proc. Sympos. Appl. Math., vol. 27, Amer. Math. Soc., 1982, p. 71-86.

[13] V. S. Vladimirov - «Construction of envelopes of holomorphy for a special kind of region », Soviet Math. Dokl. 1 (1960), p. 1039-1042. 